\title{
Existence and Hyers-Ulam stability results for a coincidence problem with applications
}

\author{
Oana Mleçniţe
}




\title{
EXISTENCE AND HYERS-ULAM STABILITY RESULTS FOR A COINCIDENCE PROBLEM WITH APPLICATIONS
}

\author{
OANA MLEŞNIŢE
}

Received 18 September, 2012

\begin{abstract}
The purpose of the work is to present some Hyers-Ulam stability results for the coincidence point problem associated to a single-valued operator problem. As an application, a Hyers-Ulam stability theorem for a initial value problem associated to a differential equation is given.
\end{abstract}

2000 Mathematics Subject Classification: 47H10; 54H25

Keywords: metric space, coincidence point, contraction, Hyers-Ulam stability

\section{INTRODUCTION}

Let $(X, d)$ be a metric space and $f: X \rightarrow X$ an operator. We denote by

$$
F i x(f):=\{x \in X \mid f(x)=x\},
$$

the fixed point set of the operator $f$. By definition, $f$ is called a weakly Picard operator if the sequence $\left(f^{n}(x)\right)_{n \in \mathbb{N}}$, of successive approximations converges for all $x \in X$ and the limit (which may depend on $x$ ) is a fixed point of $f$. For example, self Caristi type operators and self graphic contractions on complete metric spaces are examples of weakly Picard operators (see [3], [4]).

If $f$ is weakly Picard operator, then we define the operator $f^{\infty}: X \rightarrow X$ defined by $f^{\infty}(x):=\lim _{n \rightarrow \infty} f^{n}(x)$. It is clear that $f^{\infty}(X)=F i x(f)$. Moreover, $f^{\infty}$ is a set retraction of $X$ to Fix $(f)$.

If $f$ is weakly Picard operator and $F i x(f)=\left\{x^{*}\right\}$, then by definition $f$ is a Picard operator. In this case $f^{\infty}$ is the constant operator, $f^{\infty}(x)=x^{*}$, for all $x \in X$. Self Banach contractions, Kannan contractions and Ciric-Reich-Rus contractions on complete metric spaces are nice examples of Picard operators (see [3], [4]).

The following concepts are important in our consideration, see [4].

This work was possible with the financial support of the Sectoral Operational Programme for Human Resources Development 2007 - 2013, co-financed by the European Social Fund, under the project number POSDRU/107/1.5/S/76841 with the title Modern Doctoral Studies: Internationalization and Interdisciplinarity. 
Definition 1. Let $f: X \rightarrow X$ be a weakly Picard operator and $\psi: \mathbb{R}_{+} \rightarrow \mathbb{R}_{+}$an increasing function which is continuous in 0 and $\psi(0)=0$. By definition the operator $f$ is $\psi$-weakly Picard operator if

$$
d\left(x, f^{\infty}(x)\right) \leq \psi(d(x, f(x))), \text { for all } x \in X .
$$

In particular, if $\psi(t)=c \cdot t$ with $c>0$ then we say that $f$ is $c$-weakly Picard operator.

For some examples of weakly Picard operators and $\psi$-weakly Picard operators see [1].

Example 1. Let $(X, d)$ be a complete metric space and $f: X \rightarrow X$ an operator with closed graphic. We suppose that $f$ is a graphic $\alpha$-contraction, i.e.,

$$
d\left(f^{2}(x), f(x)\right) \leq \alpha d(x, f(x)), \text { for all } x \in X .
$$

Then $f$ is a $c$-weakly Picard operator, with $c=\frac{1}{1-\alpha}$.

Definition 2. Let $(x, d)$ be a metric space. A function $f: X \rightarrow X$ is a $\varphi$-contraction if $\varphi$ is increasing and $\left(\varphi^{n}(t)\right) \rightarrow 0, n \rightarrow \infty$ for all $t \geq 0$ and

$$
d(f(x), f(y)) \leq \varphi(d(x, y)), \text { for all } x, y \in X .
$$

Theorem 1 ([3]). Let $(X, d)$ be a complete metric space and $f: X \rightarrow X$ a $\varphi$ contraction. Then $f$ is a Picard operator.

Definition 3. Let $(X, d)$ be a metric space and $f: X \rightarrow X$ be an operator. By definition, the fixed point equation

$$
x=f(x)
$$

is said to be generalized Hyers-Ulam stable if there exists a function $\psi: \mathbb{R}_{+} \rightarrow \mathbb{R}_{+}$ that is increasing, continuous in 0 with $\psi(0)=0$, such that for each $\varepsilon>0$ and each solution $y^{*}$ of the inequation

$$
d(y, f(y)) \leq \varepsilon
$$

there exists a solution $x^{*}$ of the equation (1.1) such that

$$
d\left(y^{*}, x^{*}\right) \leq \psi(\varepsilon) .
$$

If $\psi(t)=c t$, for each $t \in \mathbb{R}_{+}$(for some $c>0$ ), then the equation (1.1) is said to be Hyers-Ulam stable.

Theorem 2 (see [4]). Let $(X, d)$ be a metric space. If $f: X \rightarrow X$ is a $\psi$-weakly Picard operator, then the fixed point equation (1.1) is generalized Hyers-Ulam stable. In particular, if $f$ is $c$-weakly Picard operator, then the equation (1.1) is Hyers-Ulam stable. 
Proof. Let $\varepsilon>0$ and $y^{*}$ a solution of (1.2). Since $f$ is $\psi$-weakly Picard operator, we have that

$$
d\left(x, f^{\infty}(x)\right) \leq \psi(d(x, f(x))), \text { for all } x \in X .
$$

If we take $x:=y^{*}$ and $x^{*}:=f^{\infty}(y)$, we have that $d\left(y^{*}, x^{*}\right) \leq \psi(\varepsilon)$. So, the fixed point equation (1.1) is generalized Hyers-Ulam stable.

Let $(X, d)$ and $(Y, \rho)$ be two metric spaces and $f, g: X \rightarrow Y$ two operators. Let us consider the following coincidence point problem

$$
f(x)=g(x)
$$

Definition 4 ([4]). Let $(X, d)$ and $(Y, \rho)$ be two metric spaces and $f, g: X \rightarrow Y$ be two operators. The coincidence problem (1.3) is called generalized Hyers-Ulam stable if and only if there exists $\psi: \mathbb{R}_{+} \rightarrow \mathbb{R}_{+}$that is increasing, continuous in 0 and $\psi(0)=0$ such that for every $\varepsilon>0$ and for each solution $u^{*}$ of the inequality

$$
\rho(f(u), g(u)) \leq \varepsilon
$$

there exists a solution $x^{*}$ of (1.3) such that

$$
d\left(u^{*}, x^{*}\right) \leq \psi(\varepsilon) .
$$

If there exists $c>0$ such that $\psi(t):=c t$, for each $t \in \mathbb{R}_{+}$then the coincidence point (1.3) is said to be Hyers-Ulam stable.

\section{HyERS-UlAm STABILITY FOR COINCIDENCE EQUATIONS}

Our main abstract result is an existence and Hyers-Ulam stability result for the coincidence point problem.

Theorem 3. Let $A \neq \varnothing$ be an arbitrary set and let $(M, d)$ be a metric space. Let $S, T: A \rightarrow M$ such that $S(A) \subset T(A)$ and $(T(A), d)$ is a complete subspace of $M$. Suppose that there exists a function $\varphi: \mathbb{R}_{+} \rightarrow \mathbb{R}_{+}$with $\varphi$ increasing and $\left(\varphi^{n}(t)\right) \rightarrow 0$, $n \rightarrow \infty$, for all $t \in \mathbb{R}_{+}$such that

$$
d(S x, S y) \leq \varphi(d(T x, T y)), \text { for all } x, y \in A .
$$

Then:

a) $C(S, T) \neq \varnothing$

b) If additionally, we suppose that the function $\beta(t):=t-\varphi(t)$ is increasing and bijective and there exists $\psi: \mathbb{R}_{+} \rightarrow \mathbb{R}_{+}$increasing, continuous in 0 and $\psi(0)=0$ such that

$$
d\left(y, S\left(T^{-1}(y)\right)\right) \leq \psi(d(T y, S y)), \text { for all } y \in T(A),
$$

then the coincidence point problem (1.3) is $\left(\beta^{-1} \circ \psi\right)$ - generalized Hyers-Ulam stable. 
Proof. a) The proof is organized in several steps. Let $f:=S \circ T^{-1}$. We prove:

i) $f$ is a singlevalued operator on $T(A)$;

Let $y_{1}, y_{2} \in f(x)$. We get $y_{1} \in S\left(T^{-1}(x)\right)$ and $y_{2} \in S\left(T^{-1}(x)\right)$. So exists $u_{1}, u_{2} \in T^{-1}(x)$ such that $y_{1}=S\left(u_{1}\right)$ and $y_{2}=S\left(u_{2}\right)$. Because $u_{1}, u_{2} \in T^{-1}(x)$ we have $T\left(u_{1}\right)=x$ and $T\left(u_{2}\right)=x$. Then we have:

$$
d\left(y_{1}, y_{2}\right)=d\left(S u_{1}, S u_{2}\right) \leq \varphi\left(d\left(T u_{1}, T u_{2}\right)\right)=\varphi(0) .
$$

Taking into account that $\varphi$ is increasing and $\left(\varphi^{n}(t)\right) \rightarrow 0, n \rightarrow \infty$, for all $t \in \mathbb{R}_{+}$we deduce that $\varphi(0)=0$.

We get that $d\left(y_{1}, y_{2}\right)=0$. So $y_{1}=y_{2}$ and thus $f(x)$ is a singleton.

ii) $f: T(A) \rightarrow T(A)$;

Let $x \in T(A)$. Then exists $a \in A$ such that $x=T(a)$. So we have $a \in T^{-1}(x)$ $\Longrightarrow S(a) \subseteq S\left(T^{-1}(x)\right) \Longrightarrow S(a) \subseteq f(x)$. Since $f$ is a siglevalued operator we get $S(a)=f(x) \Longrightarrow f(x)=S(a) \subseteq S(A) \subseteq T(A)$.

iii) $f: T(A) \rightarrow T(A)$ is a $\varphi$-contraction;

Let $x_{1}, x_{2} \in T(A)$ and $u_{1}, u_{2} \in A$ such that $u_{1} \in T^{-1}\left(x_{1}\right)$ and $u_{2} \in T^{-1}\left(x_{2}\right)$. Then we have:

$$
\begin{gathered}
d\left(f\left(x_{1}\right), f\left(x_{2}\right)\right)=d\left(S\left(T^{-1}\left(x_{1}\right)\right), S\left(T^{-1}\left(x_{2}\right)\right)\right)=d\left(S u_{1}, S u_{2}\right) \leq \\
\leq \varphi\left(d\left(T u_{1}, T u_{2}\right)\right)=\varphi\left(d\left(x_{1}, x_{2}\right)\right) .
\end{gathered}
$$

So $f$ is self $\varphi$-contraction on the complete metric space $(T(A), d)$.

iv) We can apply now Theorem 1 for $f$ and we deduce that $f$ is a Picard operator. So we get that there exists a unique $y^{*} \in T(A)$ such that

$$
y^{*}=f\left(y^{*}\right)=S\left(T^{-1}\left(y^{*}\right)\right) .
$$

Let $x^{*}=T^{-1}\left(y^{*}\right)$. Then $y^{*}=T\left(x^{*}\right)$ and so we get $y^{*}=S\left(x^{*}\right)$. Hence we conclude

$$
S\left(x^{*}\right)=T\left(x^{*}\right)=y^{*} .
$$

b) We prove that the coincidence point problem is generalized Hyers-Ulam stable.

Let $\varepsilon>0$ and $v^{*} \in X$ be such that $d\left(T\left(v^{*}\right), S\left(v^{*}\right)\right) \leq \varepsilon$.

If we take into account of (2.1), we have

$$
d\left(v^{*}, f\left(v^{*}\right)\right)=d\left(v^{*}, S\left(T^{-1}\left(v^{*}\right)\right)\right) \leq \psi\left(d\left(S\left(v^{*}\right), T\left(v^{*}\right)\right)\right) \leq \psi(\varepsilon) .
$$

So we get

$$
\begin{gathered}
d\left(v^{*}, y^{*}\right)=d\left(v^{*}, f\left(y^{*}\right)\right) \\
\leq d\left(f\left(v^{*}\right), v^{*}\right)+d\left(f\left(v^{*}\right), f\left(y^{*}\right)\right) \leq \psi(\varepsilon)+\varphi\left(d\left(v^{*}, y^{*}\right)\right) .
\end{gathered}
$$

Then

$$
\beta\left(\left(d\left(v^{*}, y^{*}\right)\right)\right) \leq \psi(\varepsilon)
$$


Hence we get that

$$
d\left(v^{*}, y^{*}\right) \leq\left(\beta^{-1} \circ \psi\right)(\varepsilon) .
$$

Thus, the coincidence point problem (1.3) is $\left(\beta^{-1} \circ \psi\right)$ - generalized Hyers-Ulam stable.

Remark 1. Our theorem is an extension of Goebel's Theorem (see [2]), which can be obtained from our result by taking $\varphi(t)=k t$ for each $t \in \mathbb{R}_{+}$(for some $k \in[0,1)$ ).

We will present now an application of Theorem 3.

Theorem 4. Consider the differential equation

$$
x^{\prime}=f(t, x)
$$

with the initial condition

$$
x(0)=\xi .
$$

Suppose that the function $f$ is defined in the half-plane $t \geq 0,-\infty<x<+\infty$ and satisfies following conditions:

- i) $f(t, x)$ is a continuous function of $x$ for almost all $t \geq 0$;

- ii) $f(t, x)$ is a measurable function of $t$ for all $x \in \mathbb{R}$;

- iii) Lipschitz inequality, i.e.

$$
|f(t, x)-f(t, y)| \leq L(t)|x-y|,
$$

where $L$ is locally integrable function on the interval $(0, \infty)$;

- iv) $\int_{0}^{t} f(\tau, 0) d \tau=O\left(e^{\int_{0}^{t} L(\tau) d \tau}\right)$;

- v) $f(t, \gamma u) \geq \gamma f(t, u)$ for all $\gamma \geq 1, t>0, u \in \mathbb{R}$

Then, the differential equation (2.2) has, for every $\xi \in \mathbb{R}$, a unique solution and the equation (2.2) is Hyers-Ulam stable.

Proof. Let us consider the set

$$
A=\left\{x \in C[0, \infty): x(t)=O\left(e^{\int_{0}^{t} L(\tau) d \tau}\right)\right\} .
$$

We define the operators $S, T: A \rightarrow B$ by

$$
\begin{aligned}
& (S x)(t)=\left\{\int_{0}^{t} f(\tau, x(\tau)) d \tau+\xi\right\} e^{-p \int_{0}^{t} L(\tau) d \tau}, \\
& (T x)(t)=x(t) e^{-p \int_{0}^{t} L(\tau) d \tau},
\end{aligned}
$$

where $B$ is a Banach space of bounded continuous functions on $[0, \infty)$ with the norm $\|x\|=\sup _{(0, \infty)}|x(t)|$ and $p>1$. By simple calculation we have

$$
|(S x)(t)-(S y)(t)| \leq \frac{1}{p}\|T x-T y\|
$$


and further $\|S x-S y\| \leq \frac{1}{p}\|T x-T y\|$. But $S(A) \subset T(A)$ and $T(A)$ is a complete subspace of $B$. By Theorem 3 there exists $\bar{x} \in A$ such that $S(\bar{x})=T(\bar{x})$. From this we have

$$
\bar{x}(t)=\int_{0}^{t} f(\tau, \bar{x}(\tau)) d \tau+\xi
$$

Since $T$ is a single-valued operator, $\bar{x}$ is unique. Then the differential equation (2.2) has for every $\xi \in \mathbb{R}$ a unique solution with the initial condition $\bar{x}(0)=\xi$.

Next we prove that the equation (2.2) is Hyers-Ulam stable.

We have $\left(T^{-1} y\right)(t)=y(t) \cdot e^{p \int_{0}^{t} L(\tau) d \tau}$. We prove that $d\left(y, S\left(T^{-1}(y)\right)\right) \leq \alpha d(T y, S y)$, for all $y \in T(A)$. We obtain that

$$
\begin{gathered}
S\left(T^{-1}(y)\right)(t)=\left\{\int_{0}^{t} f\left(\tau,\left(T^{-1} y\right)(\tau)\right) d \tau+\xi\right\} \cdot e^{-p \int_{0}^{t} L(\tau) d \tau}= \\
=e^{-p \int_{0}^{t} L(\tau) d \tau}\left\{\int_{0}^{t} f\left(\tau, y(\tau) e^{p \int_{0}^{t} L(\tau) d \tau}\right) d \tau+\xi\right\} .
\end{gathered}
$$

By calculations we get

$$
\begin{aligned}
& \left|y(t)-S\left(T^{-1}(y)\right)(t)\right| \\
= & \left|y(t)-e^{-p \int_{0}^{t} L(\tau) d \tau}\left\{\int_{0}^{t} f\left(\tau, y(\tau) e^{p \int_{0}^{t} L(\tau) d \tau}\right) d \tau+\xi\right\}\right| \leq \\
\leq & \left|y(t)-e^{-p \int_{0}^{t} L(\tau) d \tau}\left\{e^{p \int_{0}^{t} L(\tau) d \tau} \int_{0}^{t} f(\tau, y(\tau)) d \tau+\xi\right\}\right|= \\
= & \left|y(t)-\int_{0}^{t} f(\tau, y(\tau)) d \tau-e^{-p \int_{0}^{t} L(\tau) d \tau} \xi\right|= \\
= & \mid-e^{p \int_{0}^{t} L(\tau) d \tau}\left\{-y(t) e^{-p \int_{0}^{t} L(\tau) d \tau}+\right. \\
& \left.e^{-p \int_{0}^{t} L(\tau) d \tau} \int_{0}^{t} f(\tau, y(\tau)) d \tau+e^{-2 p \int_{0}^{t} L(\tau) d \tau} \xi\right\} \mid \leq \\
\leq & e^{p \int_{0}^{t} L(\tau) d \tau} \mid-y(t) e^{-p \int_{0}^{t} L(\tau) d \tau}+ \\
& e^{-p \int_{0}^{t} L(\tau) d \tau} \int_{0}^{t} f(\tau, y(\tau)) d \tau+e^{-p \int_{0}^{t} L(\tau) d \tau} \xi \mid= \\
= & e^{p \int_{0}^{t} L(\tau) d \tau}|(S y)(t)-(T y)(t)| .
\end{aligned}
$$

Since, all the condition of Theorem 3 hold, then the equation (2.2) is Hyers-Ulam stable. 


\section{REFERENCES}

[1] A. Chis-Novac, R. Precup, and I. A. Rus, "Data dependence of fixed points for non-self generalized contractions," Fixed Point Theory, vol. 10, no. 1, pp. 73-87, 2009.

[2] K. Goebel, "A coincidence theorem," Bull. Acad. Pol. Sci., Sér. Sci. Math. Astron. Phys., vol. 16, pp. 733-735, 1968.

[3] I. A. Rus, Generalized contractions and applications. Cluj-Napoca: Cluj University Press, 2001.

[4] I. A. Rus, "Ulam stability of ordinary differential equations," Stud. Univ. Babeş-Bolyai, Math., vol. 54, no. 4, pp. 125-133, 2009.

\section{Author's address}

\section{Oana Mleşniţe}

Department of Mathematics, Babeş-Bolyai University Cluj-Napoca,, Kogălniceanu Street No.1, 400084, Cluj-Napoca, Romania.

E-mail address: oana.mlesnite@math.ubbcluj.ro 